\title{
Erratum to: A Method of Airborne Gravimetry by Combining Strapdown Inertial and New Satellite Observations via Dynamic Networks
}

\author{
J. Skaloud, I. Colomina, M.E. Parés, M. Blázquez, J. Silva, and M. Chersich
}

Erratum to: International Association of Geodesy Symposia, 10.1007/1345_2016_229

In the references section of this chapter, author's name Mansi has been misspelled as Mansi X, instead of Mansi, A.H.

This has now been corrected.

The updated online version of the original chapter can be found under

DOI 10.1007/1345_2016_229

\section{J. Skaloud}

Geodetic Engineering Laboratory (TOPO), EPFL, Station 18, CH-1015 Lausanne, Switzerland e-mail: jan.skaloud@epfl.ch

I. Colomina • M. Blázquez

GeoNumerics, Av. de Carl Friedrich Gauss 11, E-08860 Castelldefels, Spain

M.E. Parés

CTTC, Av. de Carl Friedrich Gauss 7, E-08860 Castelldefels, Spain

J. Silva

DEIMOS Engenharia, Av. Dom João II-2 41, 1998-023 Lisboa, Portugal

M. Chersich

ESRI Italia c/o EUCENTRE, Via Ferrata 1, 27100 Pavia, Italy 